\title{
A network pharmacology study on the Tripteryguim wilfordii Hook for treatment of Crohn's disease
}

\author{
Jing Zhang ${ }^{1 \dagger}$, Qifeng Huang ${ }^{1 \dagger}$, Rui Zhao ${ }^{1}$ and Zhiyuan $\mathrm{Ma}^{2^{*}}$
}

\begin{abstract}
Background: To explore the mechanism of action of Tripterygium wilfordii Hook (TWH) in the treatment of Crohn's disease (CD) by network pharmacology.

Methods: Traditional Chinese Medicine Systems Pharmacology database and analysis platform (TCMSP) was used to obtain the active constituents and targets of TWH. "Crohn's disease" was used as a search term to search for related targets of CD from GeneCards database and OMIM database, thereby obtaining the targets of TWH against CD. The Cytoscape 3.7.1 software was used to construct a Chinese medicine compound-target network and STRING database to construct a protein-protein interaction network (PPI). The DAVID 6.8 online tool was used to perform gene ontology (GO) and kyoto encyclopedia of genes and genome (KEGG) pathway enrichment analysis of overlapping targets.

Results: The database results showed that there were 30 active ingredients (14 key active ingredients) in TWH and 36 targets were screened out for CD treatment. Network analysis indicated that main targets of main active components of TWH were target genes such as VEGFA, MAPK8 and CASP3, which are involved in the regulation of cancer pathway, TNF signal pathway, hepatitis B pathway, apoptosis pathway, NF-kappa B signal pathway and so forth.
\end{abstract}

Conclusions: TWH can play a multi-target and multi-channel synergistic treatment of CD by anti-angiogenesis, antiapoptosis, anti-inflammation and immune regulation.

Keywords: Tripterygium wilfordii hook, Crohn's disease, Network pharmacology, Anti-inflammatory agents

\section{Background}

Crohn's disease $(\mathrm{CD})$ is a chronic inflammatory bowel disease with multi-factors that genetics and environment interact to manifest the disease [1]. It is typically characterized by transmural inflammation of the intestine and could affect any part of the gastrointestinal tract from

\footnotetext{
*Correspondence: zhiyuan_ma@zju.edu.cn

${ }^{\dagger}$ Jing Zhang and Qifeng Huang contributed equally to this work.

${ }^{2}$ Department of Clinical Pharmacology, Key Laboratory of Clinical Cancer

Pharmacology and Toxicology Research of Zhejiang Province, Affiliated

Hangzhou First People's Hospital, Zhejiang University School of Medicine,

Hangzhou, Zhejiang 310006, People's Republic of China

'Department of Pharmacy, Sir Run Run Shaw Hospital, School of Medicine,

Zhejiang University, 3 East Qingchun Road, Hangzhou 310016, China
}

mouth to anus. The entire layer of the intestinal wall can be affected, which can easily lead to complications such as intestinal obstruction, intestinal perforation, and an intestinal fistula. Meanwhile, abdominal pain, diarrhea, anemia, fever, and weight loss also consist of the common symptoms of active $\mathrm{CD}$. The incidence of $\mathrm{CD}$ varies based on geographic region, ethnic groups and environment. For instance, CD's prevalence is greater in developed regions than in developing areas. However, the number of people with $\mathrm{CD}$ has been steadily increasing, particularly in Eastern countries where some districts are undergoing fast urbanization [2]. Yet the cause

(c) The Author(s). 2020 Open Access This article is licensed under a Creative Commons Attribution 4.0 International License, which permits use, sharing, adaptation, distribution and reproduction in any medium or format, as long as you give appropriate credit to the original author(s) and the source, provide a link to the Creative Commons licence, and indicate if changes were made. The images or other third party material in this article are included in the article's Creative Commons licence, unless indicated otherwise in a credit line to the material. If material is not included in the article's Creative Commons licence and your intended use is not permitted by statutory regulation or exceeds the permitted use, you will need to obtain permission directly from the copyright holder. To view a copy of this licence, visit http://creativecommons.org/licenses/by/4.0/ The Creative Commons Public Domain Dedication waiver (http://creativecommons.org/publicdomain/zero/1.0/) applies to the data made available in this article, unless otherwise stated in a credit line to the data. 
of $\mathrm{CD}$ is still unknown, and the use of drugs currently cannot completely control or relieve the symptoms. It is urgent to develop more effective therapeutic strategies for affected patients. Recent studies have revealed that patients in North America and Europe using complementary and alternative medicines as a strategy has risen from 21 to $60 \%$. And as an important part of complementary and alternative medicines, traditional Chinses medicine have been widely reported to treat inflammatory bowel disease [3].

Tripterygium wilfordii Hook (TWH) is a woody vine of the genus Tripterygium. It contains a variety of chemical components such as alkaloids, diterpenoids, triterpenoids and sesquiterpenes. As early as in Li Shizhen's book "Compendium of Materia Medical" of the sixteenth century, there are records that TWH can antirheumatism, promote blood circulation, reduce swelling, and relieve pain. In recent years, clinical studies have found that TWH can effectively induce $\mathrm{CD}$ remission and maintain remission $[4,5]$, however, the specific mechanism of TWH in CD treatment is poorly understood. Network pharmacology is an approach to drug design that encompasses systems biology, network analysis, connectivity, redundancy and pleiotropy [6]. Compared with experimental pharmacology methods, network pharmacology emphasizes multi-channel regulation of signaling pathways, therefore especially suitable for the explanation of the mechanism of traditional Chinese medicine (TCM) with multiple chemical components and molecular targets.

This study aimed to screen the bioactive components of TWH and elucidate the targets that contribute to its therapeutic effect, thereby further explaining its mechanism of action in CD treatment by network pharmacology. The study design and workflow are presented in Fig. 1.

\section{Methods}

\section{Bioactive ingredient and target identification for Tripterygium wilfordii Hook (TWH)}

The Chinese Medicine Systems Pharmacology Database and Analysis Platform (TCMSP) is a platform for integrating pharmacokinetics, medicinal chemistry, and drug-target-disease networks [7]. According to the TCMSP platform (http://lsp.nwu.edu.cn/tcmsp.php), the bioactive ingredients and targets of TWH were obtained. Bioactive ingredients $(\mathrm{OB})$ refers to the rate and extent that a drug is absorbed into the body's circulation. Druglike properties (DL) reflects the nature of a drug which has a specific functional group or contains the same or

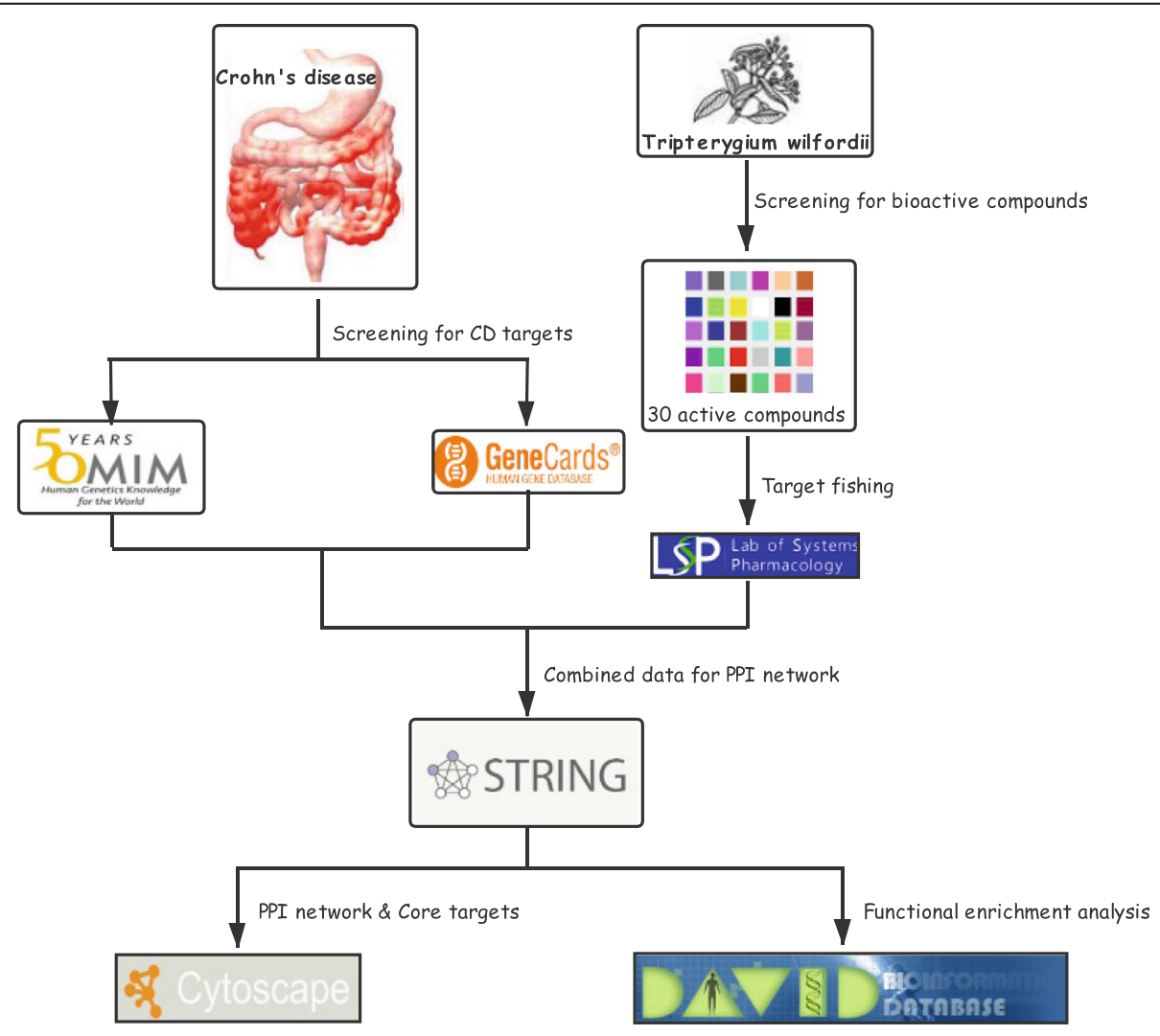

Fig. 1 Detailed design and workflow of the present study 
similar physical characteristics. The human intestinal cell line Caco-2 is a useful tool for studying the absorption and transport of drugs in intestinal epithelial cells. The drug half-life reflects the concentration of the drug in the blood or body and is an important parameter for calculating the dosing interval, the dose administered, and the drug accumulation. Bioactive ingredients were collected based on the condition that $\mathrm{OB}>30 \%, \mathrm{DL}>0.18$, Caco-2 permeability $>-0.4$, and half-life $>3 \mathrm{~h}$ as described previously [8]. Then the corresponding molecular targets of these collected active compounds were obtained by the same database.

\section{Target prediction of (TWH) in the treatment of Crohn's disease (CD)}

Search for CD related targets with "Crohn's disease" as a search term using GeneCards database (https://www. genecards.org/) and the Online Mendelian Inheritance in Man (OMIM) database (http://omim.org/). The overlapping targets from $\mathrm{CD}$ treatment and from bioactive ingredients of $\mathrm{TWH}$ then allowed identification of targets of TWH in the treatment of CD.

\section{PPI network construction}

The STRING database (https://string-db.org/) can be used to analyze the interaction between proteins and proteins [9]. In our study, the species was limited to "Homo sapiens", the lowest interaction score was set to medium confidence $(0.400)$, then discrete targets were hidden and the remaining parameters remained the default settings. Subsequently, the PPI network obtained in the STRING database was visualized and further analyzed using Cytoscape software.

\section{Core targets analysis}

Cytoscape 3.7.1 is a visualization software that provides a more intuitive analysis of the mechanism of action of drugs in treating diseases. The node represents the chemical composition and potential target of the compounds in the TCM and the edge shows the relationship between TCM component and its target. The plug-in cytoHubba of the Cytoscape software was utilized to detect the significant targets from the PPI network [10]. The top 10 nodes were shown by the maximum neighborhood component algorithm.

\section{Enrichment analysis}

The Database for Annotation, Visualization and Integrated Discovery (DAVID, https://david.ncifcrf.gov/) provides systematic, comprehensive biological annotation information for large-scale genes or proteins, and provides the most significantly enriched biological annotations [11]. Gene ontology (GO) and kyoto encyclopedia of genes and genome (KEGG) pathway enrichment of proteins in PPI network were carried out by DAVID, then the bubble plot of bioprocess and pathways were drawn by R software package.

\section{Results}

\section{Bioactive ingredients collection}

From TCMSP database, 30 bioactive components of TWH were obtained according to the OB, DL, Caco-2 and half-life values, as presented in Table 1.

\section{Target prediction of Tripterygium wilfordii Hook (TWH) in the treatment of Crohn's disease (CD)}

In our study, 62 targets of 14 bioactive components which had effect in the treatment of $\mathrm{CD}$ were obtained through TCMSP database, and 4206 genes were identified as the targets of CD from GeneCards and OMIM database in total. After mapping, 36 overlapping targets of WHT in the treatment of CD were collected.

\section{Network construction}

The compound-target interaction network was constructed by Cytoscape 3.7.1 software. The network was found to have 52 nodes, including 14 bio-active molecules, 36 common target genes, 1 drug, 1 disease, and 118 edges, as shown in Fig. 2. PPI network reflects the spatiotemporal relationship of molecules within the cell and provides valuable information about molecular mechanisms in the physiological and pathological condition [12]. The 36 common targets were then inputted into the STRING database for PPI network analysis, then visualized by Cytoscape. There were 36 nodes and 178 edges in the PPI network, as shown in Fig. 3. The average node degree of freedom is 9.89 , and the average aggregation coefficient is 0.666 . The key targets were identified via cytoHubba plugin of Cytoscape. The top 10 targets were presented in Fig. 4. Besides, among all the core targets, the darker the red, the more important it was. It suggests that target genes such as VEGFA, MAPK8, CASP3 may play crucial roles in the treatment of CD.

\section{Go and KEGG pathway enrichment analysis}

The results of DAVID database demonstrated that the target genes were mainly enriched in response to lipopolysaccharide, apoptosis, aging, drug reaction, reaction of toxic substances and so on in the biological processes (Fig. 5a); enzyme binding, transcription factor binding, RNA polymerase II core promoter proximal region sequence-specific binding, steroid binding and protein binding in molecular function (Fig. 5b); the cytosol, nucleoplasm and extracellular space in the cellular components (Fig. 5c). The pathway with the most enriched genes is the cancer pathway, with 12 genes; followed by 
Table 1 Information of 30 bioactive compounds in TWH

\begin{tabular}{|c|c|c|c|c|c|}
\hline Molecular ID & Molecular name & $\mathrm{OB}(\%)$ & Caco-2 & $\mathrm{DL}$ & $\mathrm{HL}$ \\
\hline MOL003182 & (+)-Medioresinol di-O-beta-D-glucopyranoside_qt & 60.69 & 0.45 & 0.62 & 3.05 \\
\hline MOL004443 & Zhebeiresinol & 58.72 & 0.53 & 0.19 & 3.32 \\
\hline MOL003279 & $99694-86-7$ & 75.23 & -0.13 & 0.66 & 3.71 \\
\hline MOL009386 & 3,3'-bis-(3,4-dihydro-4-hydroxy-6-methoxy)-2H-1-benzopyran & 52.11 & 0.14 & 0.54 & 3.76 \\
\hline MOL003199 & 3,3'-bis-(3,4-dihydro-4-hydroxy-6-methoxy)-2H-2-benzopyran & 61.85 & 0.02 & 0.54 & 3.97 \\
\hline MOL003189 & WILFORLIDE A & 35.66 & 0.31 & 0.72 & 4.05 \\
\hline MOL011169 & Peroxyergosterol & 44.39 & 0.86 & 0.82 & 4.06 \\
\hline MOL003187 & triptolide & 51.29 & 0.25 & 0.68 & 4.14 \\
\hline MOL003232 & Triptofordin B1 & 39.55 & 0.41 & 0.84 & 4.19 \\
\hline MOL003211 & Celaxanthin & 47.37 & 1.73 & 0.58 & 4.33 \\
\hline MOL003196 & Tryptophenolide & 48.5 & 1.11 & 0.44 & 4.42 \\
\hline MOL003188 & Tripchlorolide & 78.72 & 0.16 & 0.72 & 4.44 \\
\hline MOL003192 & Triptonide & 67.66 & 0.15 & 0.7 & 4.48 \\
\hline MOL000211 & Mairin & 55.38 & 0.73 & 0.78 & 8.87 \\
\hline MOL003224 & Tripdiotolnide & 56.4 & -0.29 & 0.67 & 4.91 \\
\hline MOL003198 & 5 alpha-Benzoyl-4 alpha-hydroxy-1 beta,8 alpha-dinicotinoyl-dihydro-agarofuran & 35.26 & -0.35 & 0.72 & 5.23 \\
\hline MOL000296 & hederagenin & 36.91 & 1.32 & 0.75 & 5.35 \\
\hline MOL000358 & beta-sitosterol & 36.91 & 1.32 & 0.75 & 5.36 \\
\hline MOL000449 & Stigmasterol & 43.83 & 1.44 & 0.76 & 5.57 \\
\hline MOL003184 & $81,827-74-9$ & 45.42 & 0.85 & 0.53 & 5.58 \\
\hline MOL007415 & [(2S)-2-[(2S)-2-(benzoylamino)-3-phenylpropanoyl]amino]-3-phenylpropyl] acetate & 58.02 & 0.32 & 0.52 & 6.03 \\
\hline MOL007535 & $\begin{array}{l}\text { (5S,8S,9S, 10R,13R,14S,17R)-17-[(1R,4R)-4-ethyl-1,5-dimethylhexyl]-10,13-dimethyl-2,4, } \\
5,7,8,9,11,12,14,15,16,17 \text {-dodecahydro-1H-cyclopenta [a]phenanthrene-3,6-dione }\end{array}$ & 33.12 & 0.9 & 0.79 & 6.56 \\
\hline MOL003266 & 21-Hydroxy-30-norhopan-22-one & 34.11 & 0.9 & 0.77 & 6.66 \\
\hline MOL003235 & Triptofordin D1 & 32 & -0.35 & 0.75 & 7.2 \\
\hline MOL003244 & Triptonide & 68.45 & 0.15 & 0.68 & 4.79 \\
\hline MOL003209 & Celallocinnine & 83.47 & 0.89 & 0.59 & 10 \\
\hline MOL000422 & kaempferol & 41.88 & 0.26 & 0.24 & 14.74 \\
\hline MOL005828 & nobiletin & 61.67 & 1 & 0.52 & 16.2 \\
\hline MOL003280 & TRIPTONOLIDE & 49.51 & 0.72 & 0.49 & 17.94 \\
\hline MOL003217 & Isoxanthohumol & 56.81 & 0.76 & 0.39 & 17.98 \\
\hline
\end{tabular}

the TNF signaling pathway, hepatitis B pathway, apoptotic pathway, NF-kappa B signaling pathway (Fig. 6a).

\section{Discussion}

$\mathrm{CD}$ is an inflammatory bowel disease of unknown etiology and currently thought to have a relationship with infection, genetics, environment and cellular immunity. At present, the drugs for clinical treatment of $\mathrm{CD}$ are mainly divided into four categories, salicylic acid preparations, steroids, immunosuppressant preparation and biological agents. But there are still many problems we should reconsider, like allergy of salicylic acid, side effects of steroids, slow and short effect of immunosuppressant, expensive and easy recurrence of drug withdrawal of biological preparations. Nowadays, the
TCM, tripterygium wilfordii glycosides derived from TWH have been widely used in immunoinflammatory diseases such as rheumatoid arthritis, lupus erythematosus, and atopic eczema [4,13-15], are gradually being used for the treatment of CD.

Abnormal neovascularization in the intestinal tract, one of the typical pathological features of CD patients, is an important factor leading to chronic and persistent intestinal inflammations [16]. Vascular endothelial growth factor A (VEGFA) is the most vital pro-angiogenic factor, which can stimulate the secretion of various inflammatory cytokines and chemokines, thus accelerate the inflammatory process. In our study, we found that VEGFA can be affected by tripolide (MOL003187). Studies have shown that VEGF levels of patients are 


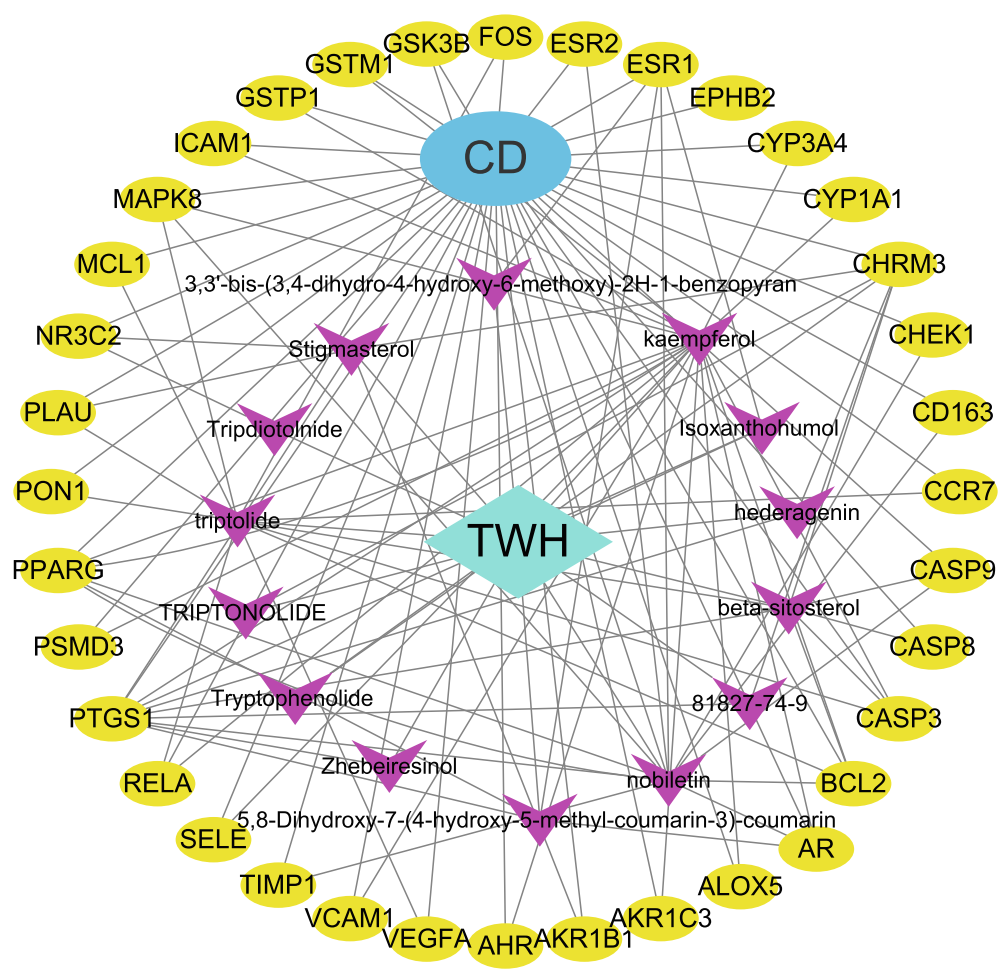

Fig. 2 TWH-CD disease network. Purple inverted triangles represent active compounds of TWH, yellow circles are potential targets of TWH for the treatment of $C D$

correlated with their CD clinical activity and appear to predict clinical response after anti-TNF- $\alpha$ treatment [17]. Some reported that VEGF levels decline faster and can maintain in low expression for a long time in those effectively treated patients [18]. In the Chinese

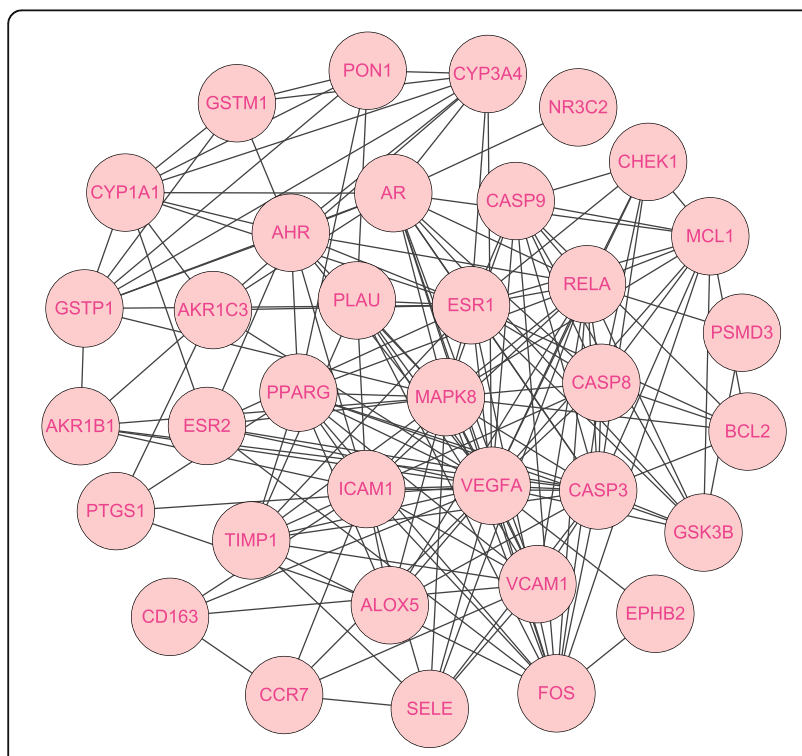

Fig. 3 Network of overlapping targets population, people with variant genotype carriers of the VEGF gene rs3025039 locus had a lower risk of nonstenotic and non-penetrating $\mathrm{CD}$. This may be related to the decrease of VEGF expression level, intestinal neovascularization and intestinal inflammatory reaction [19].

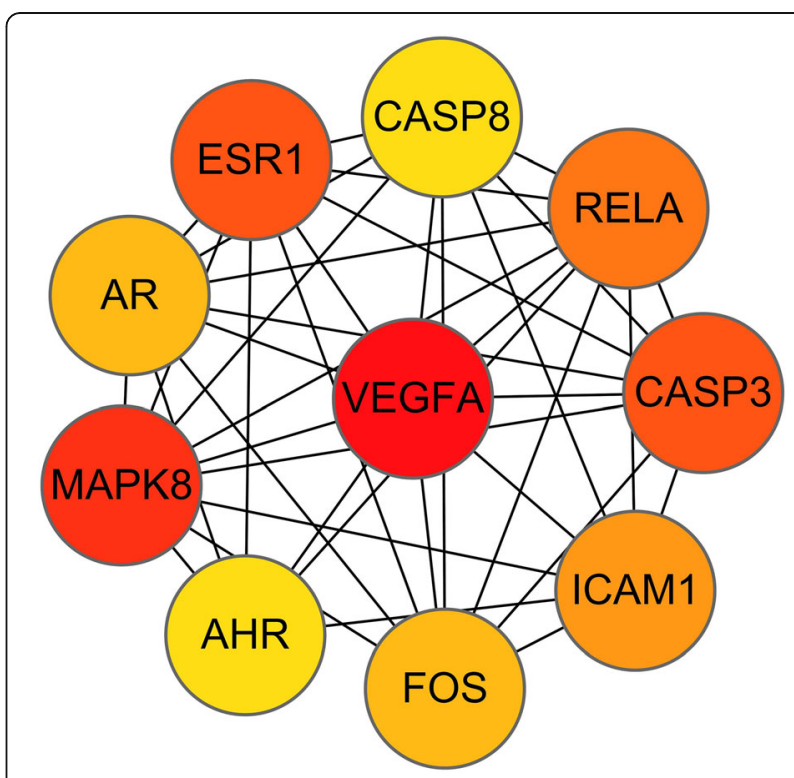

Fig. 4 Hub genes of identified targets 


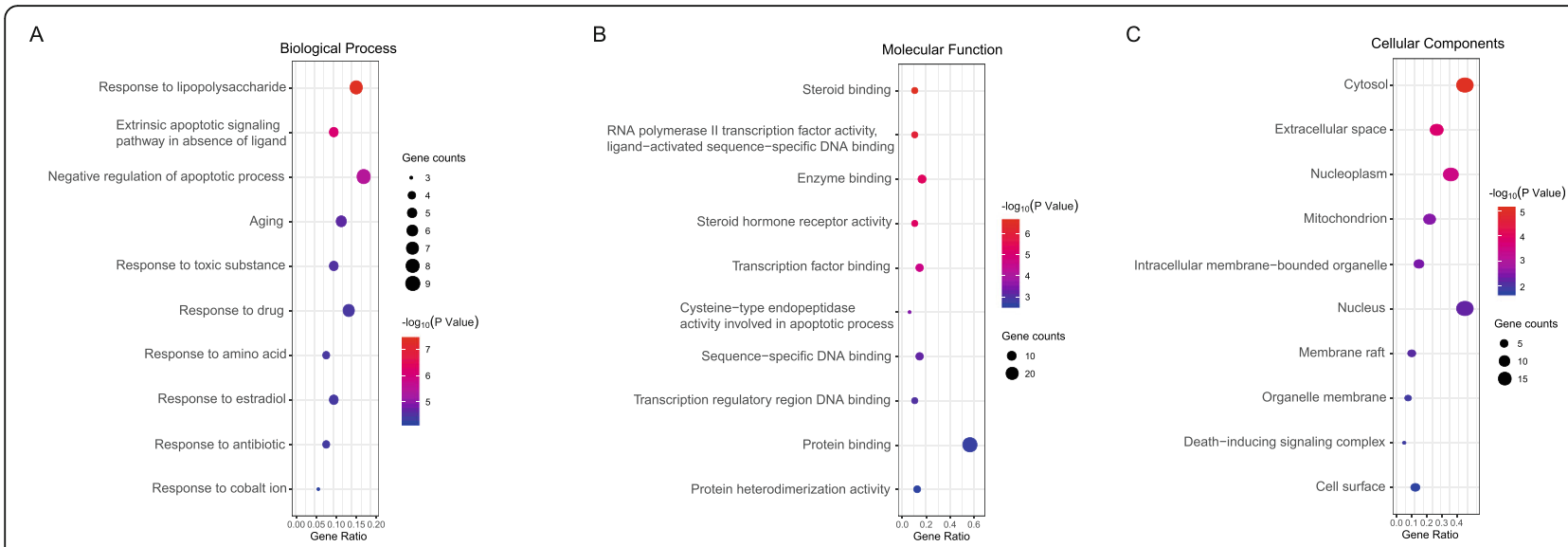

Fig. 5 Gene Ontology analysis of potential targets of TWH. a Representative bubble plots of biological functional analysis of the core targets. $\mathbf{b}$ Representative bubble plots of molecular function among candidate targets. c Representative bubble plots of cellular components of identified targets. Gene ratio $=$ count/set size

Our study also indicated that mitogen-activated protein kinase (MAPK) can be affected by kaempferol, nobiletin, and triptolide (MOL000422, MOL005828 and MOL003187). Continuous activation of extracellular regulated protein kinases, p38 kinase and c-Jun N-terminal kinase is observed in the inflammatory mucosa of CD patients [20], suggesting that MAPK transduction pathway is involved in the CD disease process. And we found that Capase-3 can be affected by kaempferol, betasitosterol, and triptolide (MOL000422, MOL000358 and MOL003187). Caspase-3 is an important gene that causes apoptosis and participates in the elevated apoptosis process of intestinal epithelial cells, which greatly impairs the epithelial barrier integrity [21]. Thus, previous and our study indicate VEGFA, MAPK8 and CASP3 may be potential targets of TWH in CD treatment, however it needs more vivo and invitro experiments evidence.

Tumor necrosis factor- $\alpha$ (TNF- $\alpha)$ is a member of a large family of proteins and receptors involved in immune regulation which interacts with two different receptors, and then initiates downstream signaling

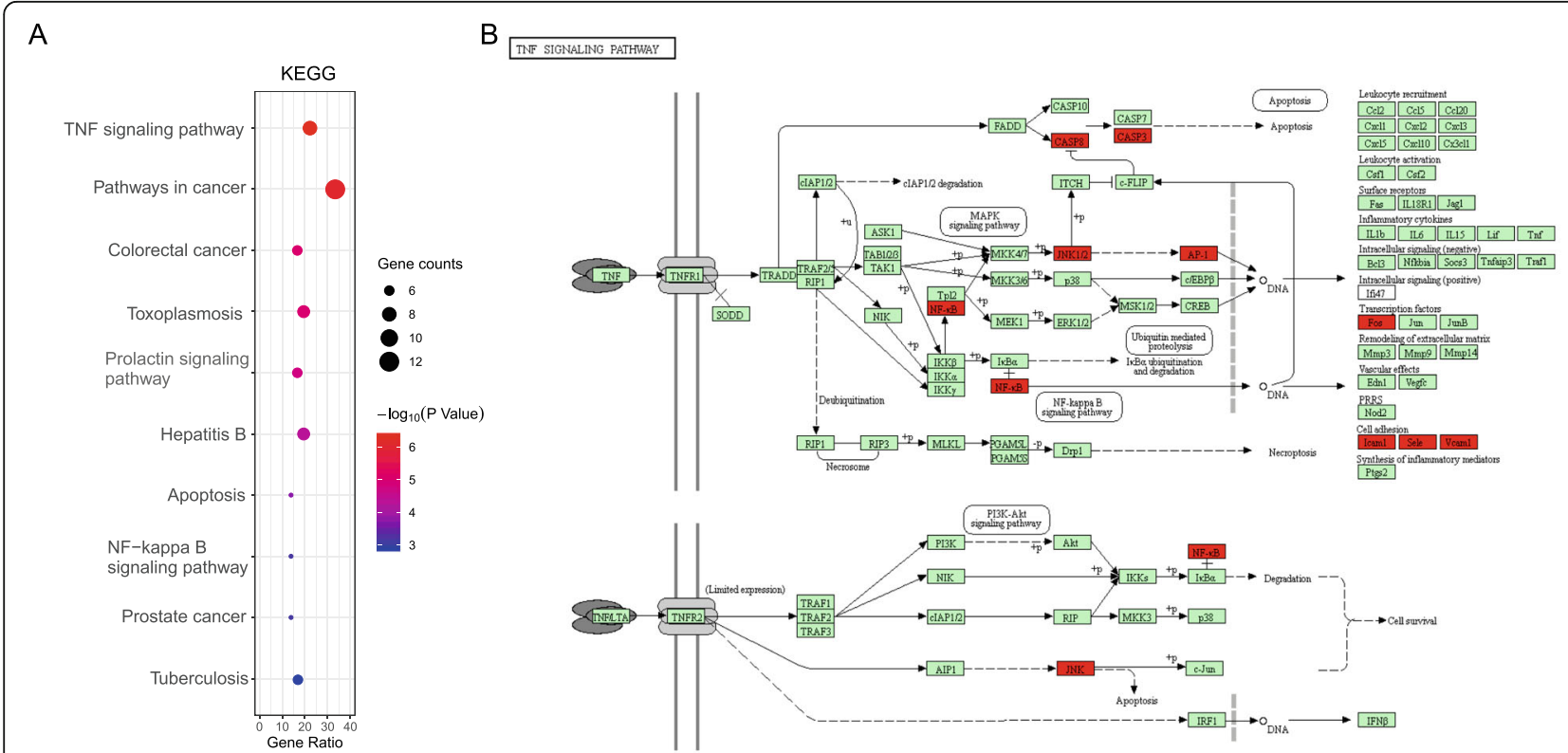

Fig. 6 Signaling pathway enrichment analysis of core targets of TWH for CD. a Representative bubble plots of the pathway enrichment analysis of the core targets. Gene ratio = count/set size. $\mathbf{b}$ Reprinted of TNF signaling pathway with permission from Kyoto Encyclopedia of Genes and Genome 
pathways to exert pro-inflammatory biological effects. In the inflammatory process of CD, TNF- $\alpha$ is an early proinflammatory cytokine and has been observed to be involved in the pathological processes in $\mathrm{CD}$ (including neutrophil accumulation, granuloma formation, increased epithelial permeability) [22]. Serum TNF- $\alpha$ is upregulated in patients with active $\mathrm{CD}$ activity compared with healthy control [23]. Triptolide, an active ingredient derived from TWH, could significantly reduce the production of TNF- $\alpha$, IFN- $\gamma$ and IL-4 in the colon [24]. In addition, it could ameliorate Th1-mediated chronic colitis and disordered immune state in IL-10(-/-) mice by inhibiting TNF-alpha/TNFR2 signal pathway [25]. NF$\kappa B$ is a widely distributed nuclear transcription factor that regulates the expression of many inflammatory mediators including TNF- $\alpha$, as presented in Fig. $6 \mathrm{~b}$. Abnormal activation of NF- $\mathrm{KB}$ starts at the initiation and progression stages of $\mathrm{CD}$ inflammation. Triptolide could inhibit the TLRs/NF- $\mathrm{kB}$ signaling pathway in vivo and in cultured colonic explants from CD patients [26].

However, due to the reported risk of toxic action on such organs as the liver, kidney, spleen, gastrointestinal tract or heart [27], application of TWH raises the question on the safety of its use in CD treatment. As toxicity was related to medication course, combined intervention, and drug dosage, the importance of follow-up animal experiments should be emphasized.

\section{Conclusions}

In this paper, network pharmacology was used to explore the active components, potential targets and mechanisms of TWH in treating CD. The results showed that 14 ingredients of TWH may play important roles in biological processes of anti-angiogenesis, anti-apoptosis, anti-inflammatory and immune regulation by acting on VEGF, MAPK8, and CASP3 through TNF- $\alpha$ and NF-kB signaling pathways. More importantly, the potential targets and biological processes mentioned above may provide valuable information for further investigation into the mechanism of TWH for treating $\mathrm{CD}$.

\footnotetext{
Abbreviations

TWH: Tripterygium wilfordii Hook; CD: Crohn's disease; TCMSP: Traditional Chinese Medicine Systems Pharmacology database and analysis platform; PPI: Protein-protein interaction network; GO: Gene ontology; KEEG: Kyoto encyclopedia of genes and genome; TCM: Traditional Chinese medicine; OMIM: Online Mendelian Inheritance in Man; DAVID: The Database for Annotation, Visualization and Integrated Discovery; VEGFA: Vascular endothelial growth factor A; MAPK: Mitogen-activated protein kinase; TNFa: Tumor necrosis factor-a
}

\section{Acknowledgements}

Not applicable.

\section{Authors' contributions}

MZY conceived and designed the experiments; ZJ, HQF, and ZR performed the research; both MZY and ZJ wrote the manuscript with input from all authors and supervised the findings of this work. All authors read and approved the final manuscript.

\section{Funding}

This study was funded by Zhejiang Provincial Natural Science Foundation of China (No. LYY18H310004).

\section{Availability of data and materials}

The datasets used and analyzed during the current study are available from the corresponding author on reasonable request.

Ethics approval and consent to participate

Not applicable.

\section{Consent for publication}

Not applicable.

\section{Competing interests}

The authors declare that they have no competing interests.

Received: 25 November 2019 Accepted: 6 March 2020

Published online: 23 March 2020

\section{References}

1. Gajendran M, Loganathan P, Catinella AP, Hashash JG. A comprehensive review and update on Crohn's disease. Dis Mon. 2018;64:20-57.

2. Torres J, Mehandru S, Colombel JF, Peyrin-Biroulet L. Crohn's disease. Lancet. 2017;389:1741-55.

3. Gao C, Liu L, Zhou Y, Bian Z, Wang S, Wang Y. Novel drug delivery systems of Chinese medicine for the treatment of inflammatory bowel disease. Chin Med. 2019;14:23.

4. Sun J, Shen X, Dong J, et al. Tripterygium wilfordii hook F as maintenance treatment for Crohn's disease. Am J Med Sci. 2015;350:345-51.

5. Zhu W, Li Y, Gong J, et al. Tripterygium wilfordii Hook. f. versus azathioprine for prevention of postoperative recurrence in patients with Crohn's disease: a randomized clinical trial. Dig Liver Dis. 2015;47:14-9.

6. Hopkins AL. Network pharmacology: the next paradigm in drug discovery. Nat Chem Biol. 2008;4:682-90.

7. Ru J, Li P, Wang J, et al. TCMSP: a database of systems pharmacology for drug discovery from herbal medicines. J Cheminform. 2014;6:13.

8. Hu W, Fu W, Wei X, Yang Y, Lu C, Liu Z. A network pharmacology study on the active ingredients and potential targets of Tripterygium wilfordii hook for treatment of rheumatoid arthritis. Evid Based Complement Alternat Med. 2019;2019:5276865.

9. von Mering C, Jensen LJ, Snel B, et al. STRING: known and predicted protein-protein associations, integrated and transferred across organisms. Nucleic Acids Res. 2005;33:D433-7.

10. Chin $\mathrm{CH}$, Chen $\mathrm{SH}, \mathrm{Wu} \mathrm{HH}, \mathrm{Ho} \mathrm{CW}$, Ko MT, Lin CY. cytoHubba: identifying hub objects and sub-networks from complex interactome. Bmc Syst Biol. 2014;8(Suppl 4):S11.

11. Dennis GJ, Sherman BT, Hosack DA, et al. DAVID: database for annotation, visualization, and integrated discovery. Genome Biol. 2003;4:P3.

12. Guo T, Ma H, Zhou Y. Bioinformatics analysis of microarray data to identify the candidate biomarkers of lung adenocarcinoma. Peerj. 2019;7:e7313.

13. Wang J, Chen N, Fang L, et al. A systematic review about the efficacy and safety of Tripterygium wilfordii Hook.f. Preparations used for the management of rheumatoid arthritis. Evid Based Complement Alternat Med. 2018. https://doi.org/10.1155/2018/1567463.

14. Liu L, Luo $Y$, Zhou M, et al. Tripterygium agents for the treatment of atopic eczema: a Bayesian analysis of randomized controlled trials. Phytomedicine. 2019;59:152914

15. Wang D, Zhang H, Liang J, et al. A long-term follow-up study of allogeneic Mesenchymal stem/stromal cell transplantation in patients with drugresistant systemic lupus Erythematosus. Stem Cell Rep. 2018;10:933-41.

16. Danese S. Inflammation and the mucosal microcirculation in inflammatory bowel disease: the ebb and flow. Curr Opin Gastroenterol. 2007:23:384-9.

17. Eder $P$, Korybalska K, Lykowska-Szuber $L$, et al. Association of serum VEGF with clinical response to anti-TNFalpha therapy for Crohn's disease. Cytokine. 2015;76:288-93.

18. Di Sabatino A, Ciccocioppo R, Benazzato L, Sturniolo GC, Corazza GR. Infliximab downregulates basic fibroblast growth factor and vascular 
endothelial growth factor in Crohn's disease patients. Aliment Pharmacol Ther. 2004;19:1019-24.

19. Pei J, Yu L, Xia X, et al. Association of vascular endothelial growth factor gene polymorphisms with Crohn's disease among Chinese patients. Zhonghua Yi Xue Yi Chuan Xue Za Zhi. 2018;35:582-6 (Article in Chinese).

20. Broom OJ, Widjaya B, Troelsen J, Olsen J, Nielsen OH. Mitogen activated protein kinases: a role in inflammatory bowel disease? Clin Exp Immunol. 2009;158:272-80.

21. Wu H, Wang L, Zhang D, et al. PRDM5 promotes the apoptosis of epithelial cells induced by IFN-gamma during Crohn's disease. Pathol Res Pract. 2017; 213:666-73.

22. Adegbola SO, Sahnan K, Warusavitarne J, Hart A, Tozer P. Anti-TNF therapy in Crohn's disease. Int J Mol Sci. 2018. https://doi.org/10.3390/ijms19082244.

23. Avdagic N, Babic N, Seremet M, et al. Tumor necrosis factor-alpha serum level in assessment of disease activity in inflammatory bowel diseases. Med Glas (Zenica). 2013;10:211-6 (View record in MEDLINE).

24. Wei X, Gong J, Zhu J, et al. Therapeutic effects of triptolide on interleukin-10 gene-deficient mice with colitis. Int Immunopharmacol. 2008;8:1808-12.

25. Wei X, Gong J, Zhu J, et al. The suppressive effect of triptolide on chronic colitis and TNF-alpha/TNFR2 signal pathway in interleukin-10 deficient mice. Clin Immunol. 2008;129:211-8.

26. Yu C, Shan T, Feng A, et al. Triptolide ameliorates Crohn's colitis is associated with inhibition of TLRs/NF-kappaB signaling pathway. Fitoterapia. 2011;82:709-15.

27. Xi C, Peng S, Wu Z, Zhou Q, Zhou J. Toxicity of triptolide and the molecular mechanisms involved. Biomed Pharmacother. 2017;90:531-41.

\section{Publisher's Note}

Springer Nature remains neutral with regard to jurisdictional claims in published maps and institutional affiliations.

Ready to submit your research? Choose BMC and benefit from:

- fast, convenient online submission

- thorough peer review by experienced researchers in your field

- rapid publication on acceptance

- support for research data, including large and complex data types

- gold Open Access which fosters wider collaboration and increased citations

- maximum visibility for your research: over $100 \mathrm{M}$ website views per year

At BMC, research is always in progress.

Learn more biomedcentral.com/submissions 